\title{
On Delay-Range-Dependent Stochastic Stability Conditions of Uncertain Neutral Delay Markovian Jump Systems
}

\author{
Xinghua Liu and Hongsheng Xi \\ Department of Automation, School of Information Science and Technology, University of Science and Technology of China, \\ Anhui 230027, China \\ Correspondence should be addressed to Xinghua Liu; salxh@mail.ustc.edu.cn
}

Received 25 March 2013; Accepted 17 June 2013

Academic Editor: Alfredo Bellen

Copyright (C) 2013 X. Liu and H. Xi. This is an open access article distributed under the Creative Commons Attribution License, which permits unrestricted use, distribution, and reproduction in any medium, provided the original work is properly cited.

The delay-range-dependent stochastic stability for uncertain neutral Markovian jump systems with interval time-varying delays is studied in this paper. The uncertainties under consideration are assumed to be time varying but norm bounded. To begin with the nominal systems, a novel augmented Lyapunov functional which contains some triple-integral terms is introduced. Then, by employing some integral inequalities and the nature of convex combination, some less conservative stochastic stability conditions are presented in terms of linear matrix inequalities without introducing any free-weighting matrices. Finally, numerical examples are provided to demonstrate the effectiveness and to show that the proposed results significantly improve the allowed upper bounds of the delay size over some existing ones in the literature.

\section{Introduction}

Time delays are frequently encountered in various engineering systems, such as chemical or process control systems, networked control systems, and manufacturing systems. To sum up, delays can appear in the state, input, or output variables (retarded systems), as well as in the state derivative (neutral systems). In fact, neutral delay systems constitute a more general class than those of the retarded type because such systems can be found in places such as population ecology [1], distributed neural networks [2], heat exchangers, and robots in contact with rigid environments [3]. Since it is shown that the existence of delays in a dynamic system may result in instability, oscillations, or poor performances [35], the stability of time-delay systems has been an important problem of recurring interest for many years. Existing results on this topic can be roughly classified into two categories, namely, delay-independent criteria [6] and delay-dependent criteria, and it is generally recognized that the latter cases are less conservative. Actually, the stability of neutral time-delay systems proves to be a more complex issue as well as singular systems [7-9] because the systems involve the derivative of the delayed state. So considerable attention has been devoted to the problem of robust delay-independent stability or delaydependent stability and stabilization via different approaches for linear neutral systems with delayed state input and parameter uncertainties. Results are mainly presented based on Lyapunov-Krasovskii (L-K) method; see, for example, [10-16] and the references therein. However, there is room for further investigation because the conservativeness of the neutral systems can be further reduced by a better technique.

On the other hand, with the development of science and technology, many practical dynamics, for example, solar thermal central receivers, robotic manipulator systems, aircraft control systems, economic systems, and so on, experience abrupt changes in their structures, whose parameters are caused by phenomena such as component failures or repairs, changes in subsystem interconnections, and sudden environmental changes. This class of systems is more appropriate to be described as Markovian jump systems (MJSs), which can be regarded as a special class of hybrid systems with finite operation modes. The system parameters jump among finite modes, and the mode switching is governed by a Markov process to represent the abrupt variation in their structures and parameters. With so many applications in engineering systems, a great deal of attention has been paid to the stability 
analysis and controller synthesis for Markovian jump systems (MJSs) in recent years. Many researchers have made a lot of progress on Markovian jump delay systems and Markovian jump control theory; see, for example, [17-23] and references therein for more details. However, a few of these papers have considered the effect of delay on the stability or stabilization for the corresponding neutral systems. Besides, to the best of the authors' knowledge, it seems that the problem of stochastic stability for neutral Markovian jumping systems with interval time-varying delays has not been fully investigated and it is very challenging. Motivated by the previous description, this paper investigates the stochastic stability of neutral Markovian jumping systems with interval timevarying delays to seek less conservative stochastic stability conditions than some previous ones.

In order to simplify the treatment of the problem, in this paper, we first investigate the nominal systems and construct a new augmented Lyapunov functional containing some triple-integral terms to reduce conservativeness. By some integral inequalities and the nature of convex combination, the delay-range-dependent stochastic stability conditions are derived for the nominal neutral systems with Markovian jump parameters and interval time-varying delays. Then, the results are extended to the corresponding uncertain case on the basis of obtained conditions. In addition, these conditions are expressed in linear matrix inequalities (LMIs), which can be easily checked by utilizing the LMI Toolbox in MATLAB. Numerical examples are given to show the effectiveness and reduced conservativeness over some previous references.

The main contributions of this paper can be summarized as follows: (1) the proposed Lyapunov functional contains some triple-integral terms which is very effective in the reduction of conservativeness, and has not been used in any of the existing literatures in the same context before; (2) the delay-range-dependent stability conditions are obtained in terms of LMIs without introducing any free-weighting matrices besides the Lyapunov matrices, which will reduce the number of variables and decrease the complexity of computation; (3) the proposed results are expressed in a new representation and proved to be less conservative than some existing ones.

The remainder of this paper is organized as follows: Section 2 contains the problem statement and preliminaries; Section 3 presents the main results; Section 4 provides a numerical example to verify the effectiveness of the results; Section 5 draws a brief conclusion.

1.1. Notations. In this paper, $\mathbb{R}^{n}$ denotes the $n$ dimensional Euclidean space and $\mathbb{R}^{m \times n}$ is for the set of all $m \times n$ matrices. The notation $X<Y(X>Y)$, where $X$ and $Y$ are both symmetric matrices, means that $X-Y$ is negative (positive) definite. $I$ denotes the identity matrix with proper dimensions. $\lambda_{\max (\min )}(A)$ is the eigenvalue of matrix $A$ with maximum (minimum) real part. For a symmetric block matrix, we use the sign $*$ to denote the terms introduced by symmetry. $\mathscr{E}$ stands for the mathematical expectation, and $\|v\|$ is the Euclidean norm of vector $v,\|v\|=\left(v^{T} v\right)^{1 / 2}$, while $\|A\|$ is spectral norm of matrix $A,\|A\|=\left[\lambda_{\max }\left(A^{T} A\right)\right]^{1 / 2}$.
$C\left([-\rho, 0], \mathbb{R}^{n}\right)$ is the space of continuous function from $[-\rho, 0]$ to $\mathbb{R}^{n}$. In addition, if not explicitly stated, matrices are assumed to have compatible dimensions.

\section{Problem Statement and Preliminaries}

Given a probability space $\{\Omega, \mathscr{F}, \mathbf{P}\}$ where $\Omega$ is the sample space, $\mathscr{F}$ is the algebra of events and $\mathbf{P}$ is the probability measure defined on $\mathscr{F} .\left\{r_{t}, t \geq 0\right\}$ is a homogeneous, finitestate Markovian process with right continuous trajectories and taking values in a finite set $S=\{1,2,3, \ldots, N\}$, with the mode transition probability matrix

$$
P\left(r_{t+\Delta t}=j \mid r_{t}=i\right)= \begin{cases}\pi_{i j} \Delta t+o(\Delta t) & i \neq j, \\ 1+\pi_{i i} \Delta t+o(\Delta t) & i=j,\end{cases}
$$

where $\Delta t>0, \lim _{\Delta t \rightarrow 0}(o(\Delta t) / \Delta t)=0$, and $\pi_{i j} \geq 0(i, j \in$ $S, i \neq j$ ) denote the transition rate from mode $i$ to $j$. For any state or mode $i \in S$, we have

$$
\pi_{i i}=-\sum_{j=1, j \neq i}^{N} \pi_{i j}
$$

In this paper, we consider the following uncertain neutral systems with Markovian jump parameters and time-varying delay over the space $\{\Omega, \mathscr{F}, \mathbf{P}\}$ as follows:

$$
\begin{aligned}
& \dot{x}(t)-C\left(r_{t}\right) \dot{x}(t-\tau)= {\left[A\left(r_{t}\right)+\Delta A\left(r_{t}\right)\right] x(t) } \\
&+\left[B\left(r_{t}\right)+\Delta B\left(r_{t}\right)\right] x(t-d(t)), \\
& x(s)=\varphi(s), \quad r_{s}=r_{0}, \quad s \in[-\rho, 0]
\end{aligned}
$$

where $x(t) \in \mathbb{R}^{n}$ is the system state and $\tau>0$ is a constant neutral delay. It is assumed that the time-varying delay $d(t)$ satisfies

$$
0<d_{1} \leq d(t) \leq d_{2}, \quad \dot{d}(t) \leq \mu,
$$

where $d_{1}<d_{2}$ and $\mu \geq 0$ are constant real values. The initial condition $\varphi(s)$ is a continuously differentiable vector-valued function. The continuous norm of $\varphi(s)$ is defined as

$$
\|\varphi\|_{c}=\max _{s \in[-\rho, 0]}|\varphi(s)|, \quad \rho=\max \left\{\tau, d_{2}\right\} ;
$$

$A\left(r_{t}\right) \in \mathbb{R}^{n \times n}, B\left(r_{t}\right) \in \mathbb{R}^{n \times n}$, and $C\left(r_{t}\right) \in \mathbb{R}^{n \times n}$ are known mode-dependent constant matrices, while $\Delta A\left(r_{t}\right) \in \mathbb{R}^{n \times n}$ and $\Delta B\left(r_{t}\right) \in \mathbb{R}^{n \times n}$ are uncertainties. For notational simplicity, when $r_{t}=i \in S, A\left(r_{t}\right), \Delta A\left(r_{t}\right), B\left(r_{t}\right), \Delta B\left(r_{t}\right)$, and $C\left(r_{t}\right)$ are, respectively, denoted as $A_{i}, \Delta A_{i}, B_{i}, \Delta B_{i}$, and $C_{i}$. Throughout this paper, the parametric matrix $\left\|C_{i}\right\|<1$ and the admissible parametric uncertainties are assumed to satisfy the following condition:

$$
\left[\begin{array}{ll}
\Delta A_{i}(t) & \Delta B_{i}(t)
\end{array}\right]=H_{i} F_{i}(t)\left[\begin{array}{ll}
E_{A i} & E_{B i}
\end{array}\right],
$$

where $H_{i}, E_{A i}$, and $E_{B i}$ are known mode-dependent constant matrices with appropriate dimensions and $F_{i}(t)$ is an unknown and time-varying matrix satisfying

$$
F_{i}^{T}(t) F_{i}(t) \leq I, \quad \forall t
$$


Particularly, when we consider $F_{i}(t)=0$, we get the nominal systems which can be described as

$$
\dot{x}(t)-C_{i} \dot{x}(t-\tau)=A_{i} x(t)+B_{i} x(t-d(t)) .
$$

Before proceeding further, the following assumptions, definitions, and lemmas need to be introduced.

Assumption 1. The system matrix $A_{i}$ (for all $i \in S$ ) is Hurwitz matrix with all the eigenvalues having negative real parts for each mode. The matrix $H_{i}$ (for all $i \in S$ ) is chosen as a full row rank matrix.

Assumption 2. The Markov process is irreducible, and the system mode $r_{t}$ is available at time $t$.

With regard to neutral systems, the operator $\mathfrak{D}$ : $C\left([-\rho, 0], \mathbb{R}^{n}\right) \rightarrow \mathbb{R}^{n}$ is defined to be

$$
\mathfrak{D}\left(x_{t}\right)=x(t)-C x(t-\tau) .
$$

Then, the stability of operator $\mathfrak{D}$ is defined as follows.

Definition 3 (see [4]). The operator $\mathfrak{D}$ is said to be stable if the homogeneous difference equation

$$
\begin{gathered}
\mathfrak{D}\left(x_{t}\right)=0, \quad t \geq 0, \\
x_{0}=\psi \in\left\{\phi \in C\left([-\rho, 0], \mathbb{R}^{n}\right): \mathfrak{D} \phi=0\right\}
\end{gathered}
$$

is uniformly asymptotically stable. In order to guarantee the stability of the operator $\mathfrak{D}$, one has assumed that $\left\|C_{i}\right\|<1$ as previosuly mentioned, which was introduced in [24].

Definition 4 (see [25]). The systems which are described in (3) are said to be stochastically stable if there exists a positive constant $\Upsilon$ such that

$$
\mathscr{E}\left\{\int_{0}^{\infty}\left\|x\left(r_{t}, t\right)\right\|^{2} d t \mid \varphi(s), s \in[-\rho, 0], r_{0}\right\}<\Upsilon .
$$

Definition 5 (see [26]). In the Euclidean space $\left\{\mathbb{R}^{n} \times S \times R^{+}\right\}$, where $x(t) \in \mathbb{R}^{n}, r_{t} \in S$, and $t \in R^{+}$, one introduces the stochastic Lyapunov-Krasovskii function of system (3) as $V\left(x(t), r_{t}=i, t>0\right)=V\left(x_{t}, i, t\right)$, the infinitesimal generator satisfying

$$
\begin{aligned}
& \mathfrak{Q} V(x(t), i, t) \\
& =\lim _{\Delta t \rightarrow 0} \frac{1}{\Delta t}\left[\mathscr{E}\left\{V\left(x(t+\Delta t), r_{t+\Delta t}, t+\Delta t\right) \mid x(t)=x, r_{t}=i\right\}\right. \\
& \quad-V(x(t), i, t)] \\
& =\frac{\partial}{\partial t} V(x(t), i, t)+\frac{\partial}{\partial x} V(x(t), i, t) \dot{x}(t) \\
& \quad+\sum_{j=1}^{N} \pi_{i j} V(x(t), j, t) .
\end{aligned}
$$

Lemma 6 (see $[27,28])$. For any constant matrix $H=H^{T}>0$ and scalars $\tau_{2}>\tau_{1}>0$ such that the following integrations are well defined, then

$$
\begin{aligned}
\text { (a) }- & \left(\tau_{2}-\tau_{1}\right) \int_{t-\tau_{2}}^{t-\tau_{1}} x^{T}(s) H x(s) d s \\
& \leq-\left[\int_{t-\tau_{2}}^{t-\tau_{1}} x^{T}(s) d s\right] H\left[\int_{t-\tau_{2}}^{t-\tau_{1}} x(s) d s\right], \\
\text { (b) }- & \frac{1}{2}\left(\tau_{2}^{2}-\tau_{1}^{2}\right) \int_{-\tau_{2}}^{-\tau_{1}} \int_{t+\theta}^{t} x^{T}(s) H x(s) d s d \theta \\
& \leq-\left[\int_{-\tau_{2}}^{-\tau_{1}} \int_{t+\theta}^{t} x^{T}(s) d s d \theta\right] H\left[\int_{-\tau_{2}}^{-\tau_{1}} \int_{t+\theta}^{t} x(s) d s d \theta\right] .
\end{aligned}
$$

Lemma 7 (see [19]). Suppose that $0 \leq \tau_{m} \leq \tau(t) \leq \tau_{M}, \Xi_{1}, \Xi_{2}$, and $\Omega$ are constant matrices of appropriate dimensions, then

$$
\left(\tau(t)-\tau_{m}\right) \Xi_{1}+\left(\tau_{M}-\tau(t)\right) \Xi_{2}+\Omega<0
$$

if and only if $\left(\tau_{M}-\tau_{m}\right) \Xi_{1}+\Omega<0$ and $\left(\tau_{M}-\tau_{m}\right) \Xi_{2}+\Omega<0$ hold.

Lemma 8 (see [29]). For given matrices $Q=Q^{T}, M$, and $N$ with appropriate dimensions,

$$
Q+M F(t) N+N^{T} F^{T}(t) M^{T}<0
$$

for all $F(t)$ satisfying $F^{T}(t) F(t) \leq I$ if and only if there exists a scalar $\delta>0$ such that

$$
Q+\delta^{-1} M M^{T}+\delta N N^{T}<0 .
$$

Lemma 9 (see [30]). Given constant matrices $\Omega_{1}, \Omega_{2}$, and $\Omega_{3}$, where $\Omega_{1}=\Omega_{1}^{T}$ and $\Omega_{2}=\Omega_{2}^{T}>0$, then $\Omega_{1}+\Omega_{3}^{T} \Omega_{2}^{-1} \Omega_{3}<0$ if and only if

$$
\left[\begin{array}{cc}
\Omega_{1} & \Omega_{3}^{T} \\
* & -\Omega_{2}
\end{array}\right]<0 \quad \text { or } \quad\left[\begin{array}{cc}
-\Omega_{2} & \Omega_{3}^{T} \\
* & \Omega_{1}
\end{array}\right]<0 .
$$

\section{Main Results}

In this section, we first consider the nominal systems described by (9) and extend to the uncertain case. The following theorems present sufficient conditions to guarantee the stochastic stability for the neutral systems with Markovian jump parameters and time-varying delays.

\subsection{Stochastic Stability for the Nominal Systems}

Theorem 10. For the given finite set $S$ of modes with transition rates matrix, scalars $d_{1}, d_{2}, \tau$, and $\mu$, the neutral systems with Markovian jump parameters and time-varying delays as described by (9) are stochastically stable if the operator $\mathfrak{D}$ is stable, and there exist symmetric positive matrices $P_{i}>0(i \in$ $S), Q_{j}>0(j=1,2,3,4,5)$, and $R_{k}>0(k=1,2, \ldots, 11)$ such that the following linear matrix inequalities hold:

$$
\begin{aligned}
& \Pi_{i 1}+\Gamma^{T} M \Gamma<0, \\
& \Pi_{i 2}+\Gamma^{T} M \Gamma<0,
\end{aligned}
$$


where

$$
\begin{aligned}
\Pi_{i 1}= & \Pi_{i 0}-2\left(e_{2}-e_{4}\right) R_{7}\left(e_{2}^{T}-e_{4}^{T}\right)-\left(e_{3}-e_{2}\right) R_{7}\left(e_{3}^{T}-e_{2}^{T}\right) \\
& -e_{8} R_{9} e_{8}^{T}-2 e_{9} R_{9} e_{9}^{T}, \\
\Pi_{i 2}= & \Pi_{i 0}-\left(e_{2}-e_{4}\right) R_{7}\left(e_{2}^{T}-e_{4}^{T}\right)-2\left(e_{3}-e_{2}\right) R_{7}\left(e_{3}^{T}-e_{2}^{T}\right) \\
& -2 e_{8} R_{9} e_{8}^{T}-e_{9} R_{9} e_{9}^{T},
\end{aligned}
$$

where

$$
\begin{aligned}
\Pi_{i 0}= & e_{1} Y e_{1}^{T}-e_{2}\left[(1-\mu) R_{3}\right] e_{2}^{T}+e_{1} P_{i} B_{i} e_{2}^{T}+e_{2} B_{i}^{T} P_{i} e_{1}^{T} \\
& +e_{1} P_{i} C_{i} e_{11}^{T}+e_{11} C_{i}^{T} P_{i} e_{1}^{T}+e_{3}\left(R_{2}+R_{3}-R_{1}\right) e_{3}^{T} \\
& -e_{4} R_{2} e_{4}^{T}+e_{5}\left(R_{5}-R_{4}\right) e_{5}^{T}-e_{6} R_{5} e_{6}^{T}-e_{7} R_{8} e_{7}^{T} \\
& -e_{10} Q_{1} e_{10}^{T}-e_{11} Q_{2} e_{11}^{T}-e_{12} Q_{3} e_{12}^{T} \\
& -\left(e_{1}-e_{10}\right) Q_{4}\left(e_{1}^{T}-e_{10}^{T}\right)-\left(e_{1}-e_{3}\right) R_{6}\left(e_{1}^{T}-e_{3}^{T}\right) \\
& -\left(\tau e_{1}-e_{12}\right) Q_{5}\left(\tau e_{1}^{T}-e_{12}^{T}\right) \\
& -\left(d_{1} e_{1}-e_{7}\right) R_{10}\left(d_{1} e_{1}^{T}-e_{7}^{T}\right) \\
& -\left(d_{12} e_{1}-e_{8}-e_{9}\right) R_{11}\left(d_{12} e_{1}^{T}-e_{8}^{T}-e_{9}^{T}\right)
\end{aligned}
$$

where $e_{i}\{i=1,2, \ldots, 12\}$ are block entry matrices; for instance,

$$
\begin{aligned}
& e_{2}^{T}=\left[\begin{array}{llllllllllll}
0 & I & 0 & 0 & 0 & 0 & 0 & 0 & 0 & 0 & 0 & 0
\end{array}\right], \\
& Y=A_{i}^{T} P_{i}+P_{i} A_{i}+\sum_{j=1}^{N} \pi_{i j} p_{j} \\
& +Q_{1}+\tau^{2} Q_{3}+R_{1}+d_{1}^{2} R_{8}+d_{12}^{2} R_{9} \\
& M=Q_{2}+\tau^{2} Q_{4}+\frac{\tau^{4}}{4} Q_{5}+R_{4} \\
& +d_{1}^{2} R_{6}+d_{12}^{2} R_{7}+\frac{d_{1}^{4}}{4} R_{10}+d_{m}^{2} R_{11}, \\
& \Gamma=\left[\begin{array}{llllllllllll}
A_{i} & B_{i} & 0 & 0 & 0 & 0 & 0 & 0 & 0 & 0 & C_{i} & 0
\end{array}\right] \text {, } \\
& d_{12}=d_{2}-d_{1}, \\
& d_{m}=\frac{1}{2}\left(d_{2}^{2}-d_{1}^{2}\right) \text {. }
\end{aligned}
$$

Proof. Construct the novel Lyapunov functional as follows:

$$
\begin{aligned}
V(x(t), i, t)= & V_{r}\left(x_{t}, i\right)+V_{\tau}\left(x_{t}, i\right)+V_{d 1}\left(x_{t}, i\right) \\
& +V_{d 2}\left(x_{t}, i\right)+V_{d 3}\left(x_{t}, i\right),
\end{aligned}
$$

where

$$
\begin{aligned}
& V_{r}\left(x_{t}, i\right)=x^{T}(t) P_{i} x(t), \\
& V_{\tau}\left(x_{t}, i\right)=\int_{t-\tau}^{t} x^{T}(s) Q_{1} x(s) d s+\int_{t-\tau}^{t} \dot{x}^{T}(s) Q_{2} \dot{x}(s) d s \\
& +\int_{-\tau}^{0} \int_{t+\theta}^{t} x^{T}(s)\left[\tau Q_{3}\right] x(s) d s d \theta \\
& +\int_{-\tau}^{0} \int_{t+\theta}^{t} \dot{x}^{T}(s)\left[\tau Q_{4}\right] \dot{x}(s) d s d \theta \\
& +\int_{-\tau}^{0} \int_{\theta}^{0} \int_{t+\lambda}^{t} \dot{x}^{T}(s)\left[\frac{\tau^{2}}{2} Q_{5}\right] \dot{x}(s) d s d \lambda d \theta, \\
& V_{d 1}\left(x_{t}, i\right)=\int_{t-d_{1}}^{t} x^{T}(s) R_{1} x(s) d s+\int_{t-d_{2}}^{t-d_{1}} x^{T}(s) R_{2} x(s) d s \\
& +\int_{t-d(t)}^{t-d_{1}} x^{T}(s) R_{3} x(s) d s \\
& +\int_{t-d_{1}}^{t} \dot{x}^{T}(s) R_{4} \dot{x}(s) d s \\
& +\int_{t-d_{2}}^{t-d_{1}} \dot{x}^{T}(s) R_{5} \dot{x}(s) d s, \\
& V_{d 2}\left(x_{t}, i\right)=\int_{-d_{1}}^{0} \int_{t+\theta}^{t} \dot{x}^{T}(s)\left[d_{1} R_{6}\right] \dot{x}(s) d s d \theta \\
& +\int_{-d_{2}}^{-d_{1}} \int_{t+\theta}^{t} \dot{x}^{T}(s)\left[d_{12} R_{7}\right] \dot{x}(s) d s d \theta \\
& +\int_{-d_{1}}^{0} \int_{t+\theta}^{t} x^{T}(s)\left[d_{1} R_{8}\right] x(s) d s d \theta \\
& +\int_{-d_{2}}^{-d_{1}} \int_{t+\theta}^{t} x^{T}(s)\left[d_{12} R_{9}\right] x(s) d s d \theta \\
& V_{d 3}\left(x_{t}, i\right)=\int_{-d_{1}}^{0} \int_{\theta}^{0} \int_{t+\lambda}^{t} \dot{x}^{T}(s)\left[\frac{d_{1}^{2}}{2} R_{10}\right] \dot{x}(s) d s d \lambda d \theta \\
& +\int_{-d_{2}}^{-d_{1}} \int_{\theta}^{0} \int_{t+\lambda}^{t} \dot{x}^{T}(s)\left[d_{m} R_{11}\right] \dot{x}(s) d s d \lambda d \theta \text {. }
\end{aligned}
$$

From Definition 5, taking $\mathbb{Q}$ as its infinitesimal generator along the trajectory of system (9), then from (23) and (24) we get the following equalities and inequalities:

$$
\begin{aligned}
\mathfrak{L} V(x(t), i, t)= & \mathfrak{Q} V_{r}\left(x_{t}, i\right)+\mathfrak{Q} V_{\tau}\left(x_{t}, i\right)+\mathfrak{Q} V_{d 1}\left(x_{t}, i\right) \\
+ & \mathbb{2} V_{d 2}\left(x_{t}, i\right)+\mathfrak{Q} V_{d 3}\left(x_{t}, i\right), \\
\mathfrak{Q} V_{r}\left(x_{t}, i\right)=2\left[x^{T}(t) A_{i}^{T}+x^{T}(t-d(t)) B_{i}^{T}\right. & \left.+\dot{x}^{T}(t-\tau) C_{i}^{T}\right] P_{i} x(t) \\
+ & \sum_{j=1}^{N} \pi_{i j} x^{T}(t) P_{j} x(t),
\end{aligned}
$$




$$
\begin{aligned}
\mathfrak{S} V_{\tau}\left(x_{t}, i\right)= & x^{T}(t)\left[Q_{1}+\tau^{2} Q_{3}\right] x(t) \\
& +\dot{x}^{T}(t)\left[Q_{2}+\tau^{2} Q_{4}+\frac{\tau^{4}}{4} Q_{5}\right] \dot{x}(t) \\
& -x^{T}(t-\tau) Q_{1} x(t-\tau) \\
& -\dot{x}^{T}(t-\tau) Q_{2} \dot{x}(t-\tau) \\
& -\int_{t-\tau}^{t} x^{T}(s)\left[\tau Q_{3}\right] x(s) d s \\
& -\int_{t-\tau}^{t} \dot{x}^{T}(s)\left[\tau Q_{4}\right] \dot{x}(s) d s \\
& -\int_{-\tau}^{0} \int_{t+\theta}^{t} \dot{x}(s)\left[\frac{\tau^{2}}{2} Q_{5}\right] \dot{x}(s) d s d \theta,
\end{aligned}
$$

$\mathfrak{S} V_{d 1}\left(x_{t}, i\right)=x^{T}(t) R_{1} x(t)+\dot{x}^{T}(t) R_{4} \dot{x}(t)$

$$
\begin{aligned}
& +x^{T}\left(t-d_{1}\right)\left[R_{2}+R_{3}-R_{1}\right] x\left(t-d_{1}\right) \\
& +\dot{x}^{T}\left(t-d_{1}\right)\left[R_{5}-R_{4}\right] \dot{x}\left(t-d_{1}\right) \\
& -\dot{x}^{T}\left(t-d_{2}\right) R_{5} \dot{x}\left(t-d_{2}\right) \\
& -x^{T}\left(t-d_{2}\right) R_{2} x\left(t-d_{2}\right) \\
& -(1-\dot{d}(t)) x^{T}(t-d(t)) R_{3} x(t-d(t)),
\end{aligned}
$$$$
\mathfrak{Q} V_{d 2}\left(x_{t}, i\right)=x^{T}(t)\left[d_{1}^{2} R_{8}+d_{12}^{2} R_{9}\right] x(t)
$$$$
+\dot{x}^{T}(t)\left[d_{1}^{2} R_{6}+d_{12}^{2} R_{7}\right] \dot{x}(t)
$$$$
-\int_{t-d_{1}}^{t} \dot{x}^{T}(s)\left[d_{1} R_{6}\right] \dot{x}(s) d s
$$$$
-\int_{t-d_{2}}^{t-d_{1}} \dot{x}^{T}(s)\left[d_{12} R_{7}\right] \dot{x}(s) d s
$$$$
-\int_{t-d_{1}}^{t} x^{T}(s)\left[d_{1} R_{8}\right] x(s) d s
$$$$
-\int_{t-d_{2}}^{t-d_{1}} x^{T}(s)\left[d_{12} R_{9}\right] x(s) d s,
$$$$
\mathfrak{S} V_{d 3}\left(x_{t}, i\right)=\dot{x}^{T}(t)\left[\frac{d_{1}^{4}}{4} R_{10}+d_{m}^{2} R_{11}\right] \dot{x}(t)
$$$$
-\int_{-d_{1}}^{0} \int_{t+\theta}^{t} \dot{x}^{T}(s)\left[\frac{d_{1}^{2}}{2} R_{10}\right] \dot{x}(s) d s d \theta
$$$$
-\int_{-d_{2}}^{-d_{1}} \int_{t+\theta}^{t} \dot{x}^{T}(s)\left[d_{m} R_{11}\right] \dot{x}(s) d s d \theta .
$$

\section{Let us define}

$\xi(t)$

$$
\begin{gathered}
=\operatorname{col}\left\{x(t) x(t-d(t)) x\left(t-d_{1}\right) x\left(t-d_{2}\right) \dot{x}\left(t-d_{1}\right)\right. \\
\dot{x}\left(t-d_{2}\right) \int_{t-d_{1}}^{t} x(s) d s \int_{t-d(t)}^{t-d_{1}} x(s) d s \\
\left.\int_{t-d_{2}}^{t-d(t)} x(s) d s x(t-\tau) \dot{x}(t-\tau) \int_{t-\tau}^{t} x(s) d s\right\} .
\end{gathered}
$$

Applying (a) of Lemma 6, we obtain

$$
\begin{aligned}
& -\int_{t-\tau}^{t} x^{T}(s)\left[\tau Q_{3}\right] x(s) d s \\
& \quad \leq-\left[\int_{t-\tau}^{t} x^{T}(s) d s\right] Q_{3}\left[\int_{t-\tau}^{t} x(s) d s\right] \\
& \quad=-\xi^{T}(t) e_{12} Q_{3} e_{12}^{T} \xi(t) .
\end{aligned}
$$

Following the same procedure, we also obtain the inequalities as follows:

$$
\begin{aligned}
& -\int_{t-\tau}^{t} \dot{x}^{T}(s)\left[\tau Q_{4}\right] \dot{x}(s) d s \\
& \quad \leq-\xi^{T}(t)\left(e_{1}-e_{10}\right) Q_{4}\left(e_{1}^{T}-e_{10}^{T}\right) \xi(t), \\
& -\int_{t-d_{1}}^{t} \dot{x}^{T}(s)\left[d_{1} R_{6}\right] \dot{x}(s) d s \\
& \quad \leq-\xi^{T}(t)\left(e_{1}-e_{3}\right) R_{6}\left(e_{1}^{T}-e_{3}^{T}\right) \xi(t), \\
& -\int_{t-d_{1}}^{t} x^{T}(s)\left[d_{1} R_{8}\right] x(s) d s \\
& \quad \leq-\left[\int_{t-d_{1}}^{t} x^{T}(s) d s\right] R_{8}\left[\int_{t-d_{1}}^{t} x(s) d s\right] .
\end{aligned}
$$

Applying (b) of Lemma 6, we have

$$
\begin{aligned}
& -\int_{-\tau}^{0} \int_{t+\theta}^{t} \dot{x}^{T}(s)\left[\frac{\tau^{2}}{2} Q_{5}\right] \dot{x}(s) d s d \theta \\
& \quad \leq-\left[\int_{-\tau}^{0} \int_{t+\theta}^{t} \dot{x}^{T}(s) d s d \theta\right] Q_{5}\left[\int_{-\tau}^{0} \int_{t+\theta}^{t} \dot{x}(s) d s d \theta\right] \\
& =-\left[\tau x^{T}(t)-\int_{t-\tau}^{t} x^{T}(s) d s\right] Q_{5}\left[\tau x(t)-\int_{t-\tau}^{t} x(s) d s\right] \\
& =-\xi^{T}(t)\left(\tau e_{1}-e_{12}\right) Q_{5}\left(\tau e_{1}^{T}-e_{12}^{T}\right) \xi(t) .
\end{aligned}
$$


Then the following inequalities are obtained by the same technique:

$$
\begin{aligned}
& -\int_{-d_{1}}^{0} \int_{t+\theta}^{t} \dot{x}^{T}(s)\left[\frac{d_{1}^{2}}{2} R_{10}\right] \dot{x}(s) d s d \theta \\
& \quad \leq-\xi^{T}(t)\left(d_{1} e_{1}-e_{7}\right) R_{10}\left(d_{1} e_{1}^{T}-e_{7}^{T}\right) \xi(t), \\
& -\int_{-d_{2}}^{-d_{1}} \int_{t+\theta}^{t} \dot{x}^{T}(s)\left[d_{m} R_{11}\right] \dot{x}(s) d s d \theta \\
& \quad \leq-\xi^{T}(t)\left(d_{12} e_{1}-e_{8}-e_{9}\right) R_{11}\left(d_{12} e_{1}^{T}-e_{8}^{T}-e_{9}^{T}\right) \xi(t) .
\end{aligned}
$$

Let $\lambda(t)=\left(d(t)-d_{1}\right) / d_{12}$, then we have

$$
\begin{aligned}
& -\int_{t-d_{2}}^{t-d_{1}} \dot{x}^{T}(s)\left[d_{12} R_{7}\right] \dot{x}(s) d s \\
& =-d_{12} \int_{t-d_{2}}^{t-d(t)} \dot{x}^{T}(s) R_{7} \dot{x}(s) d s \\
& -d_{12} \int_{t-d(t)}^{t-d_{1}} \dot{x}^{T}(s) R_{7} \dot{x}(s) d s \\
& =-\left(d_{2}-d(t)\right) \int_{t-d_{2}}^{t-d(t)} \dot{x}^{T}(s) R_{7} \dot{x}(s) d s \\
& -\left(d(t)-d_{1}\right) \int_{t-d_{2}}^{t-d(t)} \dot{x}^{T}(s) R_{7} \dot{x}(s) d s \\
& -\left(d(t)-d_{1}\right) \int_{t-d(t)}^{t-d_{1}} \dot{x}^{T}(s) R_{7} \dot{x}(s) d s \\
& -\left(d_{2}-d(t)\right) \int_{t-d(t)}^{t-d_{1}} \dot{x}^{T}(s) R_{7} \dot{x}(s) d s \\
& =-\xi^{T}(t)\left(e_{2}-e_{4}\right) R_{7}\left(e_{2}^{T}-e_{4}^{T}\right) \xi(t) \\
& -\frac{d(t)-d_{1}}{d_{2}-d(t)} \xi^{T}(t)\left(e_{2}-e_{4}\right) R_{7}\left(e_{2}^{T}-e_{4}^{T}\right) \xi(t) \\
& -\xi^{T}(t)\left(e_{3}-e_{2}\right) R_{7}\left(e_{3}^{T}-e_{2}^{T}\right) \xi(t) \\
& -\frac{d_{2}-d(t)}{d(t)-d_{1}} \xi^{T}(t)\left(e_{3}-e_{2}\right) R_{7}\left(e_{3}^{T}-e_{2}^{T}\right) \xi(t) \\
& \leq-\xi^{T}(t)\left(e_{2}-e_{4}\right) R_{7}\left(e_{2}^{T}-e_{4}^{T}\right) \xi(t) \\
& -\lambda(t) \xi^{T}(t)\left(e_{2}-e_{4}\right) R_{7}\left(e_{2}^{T}-e_{4}^{T}\right) \xi(t) \\
& -\xi^{T}(t)\left(e_{3}-e_{2}\right) R_{7}\left(e_{3}^{T}-e_{2}^{T}\right) \xi(t) \\
& -(1-\lambda(t)) \xi^{T}(t)\left(e_{3}-e_{2}\right) R_{7}\left(e_{3}^{T}-e_{2}^{T}\right) \xi(t) \\
& =-(1+\lambda(t)) \xi^{T}(t)\left(e_{2}-e_{4}\right) R_{7}\left(e_{2}^{T}-e_{4}^{T}\right) \xi(t) \\
& -(2-\lambda(t)) \xi^{T}(t)\left(e_{3}-e_{2}\right) R_{7}\left(e_{3}^{T}-e_{2}^{T}\right) \xi(t) .
\end{aligned}
$$

Consistent with the technique of (33), we obtain

$$
\begin{aligned}
-\int_{t-d_{2}}^{t-d_{1}} x^{T}(s)\left[d_{12} R_{9}\right] x(s) d s & \\
\leq & -(2-\lambda(t)) \xi^{T}(t)\left(e_{8} R_{9} e_{8}^{T}\right) \xi(t) \\
& \quad-(1+\lambda(t)) \xi^{T}(t)\left(e_{9} R_{9} e_{9}^{T}\right) \xi(t),
\end{aligned}
$$

considering

$$
\begin{aligned}
\dot{x}^{T}(t) M \dot{x}(t)= & \left(A_{i} x(t)+B_{i} x(t-d(t))+C_{i} \dot{x}(t-\tau)\right)^{T} \\
& \times M\left(A_{i} x(t)+B_{i} x(t-d(t))+C_{i} \dot{x}(t-\tau)\right) \\
= & \xi^{T}(t) \Gamma^{T} M \Gamma \xi(t),
\end{aligned}
$$

where $M, \Gamma$ have been defined as before.

We take the previous equalities and inequalities (26)-(35) into (25); thus, we finally get

$$
\begin{aligned}
\mathfrak{Q V}(x(t), i, t) \leq & \xi^{T}(t)\left[\lambda(t) \Pi_{i 1}+(1-\lambda(t)) \Pi_{i 2}+\Gamma^{T} M \Gamma\right] \\
& \times \xi(t) .
\end{aligned}
$$

Since $0 \leq \lambda(t) \leq 1$, by utilizing Lemma 7, we know that $\lambda(t) \Pi_{i 1}+(1-\lambda(t)) \Pi_{i 2}+\Gamma^{T} M \Gamma<0$ is equivalent to (19). So we choose

$$
\beta=\max _{i \in S, \lambda(t) \in[0,1]} \lambda_{\max }\left[\lambda(t) \Pi_{i 1}+(1-\lambda(t)) \Pi_{i 2}+\Gamma^{T} M \Gamma\right] .
$$

Then $\beta<0$ and

$$
\mathfrak{Q} V(x(t), i, t) \leq \beta\|\xi(t)\|^{2} \leq \beta\|x(t)\|^{2} .
$$

According to (38), from Dynkin's formula [31], we obtain

$$
\mathscr{E}\{V(x(t), i, t)\}-V\left(x_{0}, r_{0}\right) \leq \beta \mathscr{E}\left\{\int_{0}^{t}\|x(s)\|^{2} d s\right\} .
$$

Let $t \rightarrow \infty$, then we have

$$
\lim _{t \rightarrow \infty} \mathscr{E}\left\{\int_{0}^{t}\|x(s)\|^{2} d s\right\} \leq(-\beta)^{-1} V\left(x_{0}, r_{0}\right)
$$

From Definition 4, we know that the systems described by (9) are stochastically stable. This completes the proof.

Remark 11. Theorem 10 provides a delay-range-dependent stochastic stability criterion for nominal neutral systems with interval time-varying delays and Markovian jump parameters as described by (9). By utilizing a new Lyapunov functional, the less conservative criterion is obtained in terms of LMIs and it can be verified in Section 4 .

Remark 12. In the same context of the stochastic stability for neutral systems with Markovian jumping parameters 
and time-varying delays, the type of augmented Lyapunov functional has not been used in any of the existing literatures. Compared with the existing Lyapunov functional, the proposed one (23) contains some triple-integral terms, which is very effective in the reduction of conservativeness in [28]. Besides, the information on the lower bound of the delay is sufficiently used in the Lyapunov functional by introducing the terms, such as $\int_{t-d_{2}}^{t-d_{1}} x^{T}(s) R_{2} x(s) d s$ and $\int_{t-d(t)}^{t-d_{1}} x^{T}(s) R_{3} x(s) d s$

In many circumstances, the information on the delay derivative may not be available. That is, $\mu$ is usually unknown in the real systems. So we give the following result as a corollary which can be obtained from Theorem 10 by setting $R_{3}=0$.

Corollary 13. For the given finite set $S$ of modes with transition rates matrix, scalars $d_{1}, d_{2}$, and $\tau$, the neutral systems with Markovian jump parameters and time-varying delays as described by (9) are stochastically stable if the operator $\mathfrak{D}$ is stable, and there exist symmetric positive matrices $P_{i}>0(i \in$ $S), Q_{j}>0(j=1,2,3,4,5)$, and $R_{k}>0(k=1,2,4,5, \ldots, 11)$ such that the following linear matrix inequalities hold:

$$
\begin{aligned}
& \widetilde{\Pi}_{i 1}+\Gamma^{T} M \Gamma<0, \\
& \widetilde{\Pi}_{i 2}+\Gamma^{T} M \Gamma<0,
\end{aligned}
$$

where

$$
\begin{aligned}
\widetilde{\Pi}_{i 1}= & \widetilde{\Pi}_{i 0}-2\left(e_{2}-e_{4}\right) R_{7}\left(e_{2}^{T}-e_{4}^{T}\right) \\
& -\left(e_{3}-e_{2}\right) R_{7}\left(e_{3}^{T}-e_{2}^{T}\right)-e_{8} R_{9} e_{8}^{T}-2 e_{9} R_{9} e_{9}^{T}, \\
\widetilde{\Pi}_{i 2}= & \widetilde{\Pi}_{i 0}-\left(e_{2}-e_{4}\right) R_{7}\left(e_{2}^{T}-e_{4}^{T}\right) \\
& -2\left(e_{3}-e_{2}\right) R_{7}\left(e_{3}^{T}-e_{2}^{T}\right)-2 e_{8} R_{9} e_{8}^{T}-e_{9} R_{9} e_{9}^{T},
\end{aligned}
$$

where

$$
\begin{aligned}
\widetilde{\Pi}_{i 0}= & e_{1} Y e_{1}^{T}+e_{1} P_{i} B_{i} e_{2}^{T}+e_{2} B_{i}^{T} P_{i} e_{1}^{T}+e_{1} P_{i} C_{i} e_{11}^{T}+e_{11} C_{i}^{T} P_{i} e_{1}^{T} \\
& +e_{3}\left(R_{2}-R_{1}\right) e_{3}^{T}-e_{4} R_{2} e_{4}^{T}+e_{5}\left(R_{5}-R_{4}\right) e_{5}^{T}-e_{6} R_{5} e_{6}^{T} \\
& -e_{7} R_{8} e_{7}^{T}-e_{10} Q_{1} e_{10}^{T}-e_{11} Q_{2} e_{11}^{T}-e_{12} Q_{3} e_{12}^{T} \\
& -\left(e_{1}-e_{10}\right) Q_{4}\left(e_{1}^{T}-e_{10}^{T}\right)-\left(e_{1}-e_{3}\right) R_{6}\left(e_{1}^{T}-e_{3}^{T}\right) \\
& -\left(\tau e_{1}-e_{12}\right) Q_{5}\left(\tau e_{1}^{T}-e_{12}^{T}\right) \\
& -\left(d_{1} e_{1}-e_{7}\right) R_{10}\left(d_{1} e_{1}^{T}-e_{7}^{T}\right) \\
& -\left(d_{12} e_{1}-e_{8}-e_{9}\right) R_{11}\left(d_{12} e_{1}^{T}-e_{8}^{T}-e_{9}^{T}\right),
\end{aligned}
$$

and other notations are the same as Theorem 10.

3.2. Stochastic Stability for the Uncertain Neutral Markovian Jump Systems. In this subsection, we consider the uncertain case which can be described by (3). Based on Theorem 10, we obtain the following theorem to guarantee the stochastic stability for the uncertain neutral systems with interval timevarying delays and Markovian jump parameters.

Theorem 14. For the given finite set $S$ of modes with transition rates matrix, scalars $d_{1}, d_{2}, \tau$, and $\mu$, the uncertain neutral systems with Markovian jump parameters and time-varying delays as described by (3) are stochastically stable if the operator D is stable, and there exist scalars $\delta_{1}>0, \delta_{2}>0$, symmetric positive matrices $P_{i}>0(i \in S), Q_{j}>0(j=1,2,3,4,5)$, and $R_{k}>0(k=1,2, \ldots, 11)$ such that the following matrix inequalities hold:

$$
\begin{gathered}
{\left[\begin{array}{cc}
\Pi_{i 1}+\frac{1}{\delta_{1}} e_{1} P_{i} H_{i} H_{i}^{T} P_{i} e_{1}^{T}+\delta_{1} \varepsilon \varepsilon^{T} & \left(\Gamma^{T}+\frac{1}{\delta_{1}} e_{1} P_{i} H_{i} H_{i}^{T}\right) M \\
* & \frac{1}{\delta_{1}} M H_{i} H_{i}^{T} M-M
\end{array}\right]<0,} \\
* \begin{array}{cc}
\Pi_{i 2}+\frac{1}{\delta_{2}} e_{1} P_{i} H_{i} H_{i}^{T} P_{i} e_{1}^{T}+\delta_{2} \varepsilon \varepsilon^{T} & \left(\begin{array}{c}
\left.\Gamma^{T}+\frac{1}{\delta_{2}} e_{1} P_{i} H_{i} H_{i}^{T}\right) M \\
\frac{1}{\delta_{2}} M H_{i} H_{i}^{T} M-M
\end{array}\right]<0,
\end{array}
\end{gathered}
$$

where $\varepsilon^{T}=E_{A i} e_{1}^{T}+E_{B i} e_{2}^{T}, \Pi_{i 1}, \Pi_{i 2}, \Gamma$, and $M$ have been defined in Theorem 10.

Proof. On the basis of Theorem 10, we directly replace $A_{i}$ and $B_{i}$ with $A_{i}+\Delta A_{i}(t), B_{i}+\Delta B_{i}(t)$ and obtain

$$
\begin{aligned}
& \Pi_{i 1}(t)+\Gamma^{T}(t) M \Gamma(t)<0, \\
& \Pi_{i 2}(t)+\Gamma^{T}(t) M \Gamma(t)<0,
\end{aligned}
$$

where

$$
\begin{aligned}
\Pi_{i 1}(t)= & \Pi_{i 1}+e_{1}\left[\Delta A_{i}^{T}(t) P_{i}+P_{i} \Delta A_{i}(t)\right] e_{1}^{T} \\
& +e_{1} P_{i} \Delta B_{i}(t) e_{2}^{T}+e_{2} \Delta B_{i}^{T}(t) P_{i} e_{1}^{T}, \\
\Pi_{i 2}(t)= & \Pi_{i 2}+e_{1}\left[\Delta A_{i}^{T}(t) P_{i}+P_{i} \Delta A_{i}(t)\right] e_{1}^{T} \\
& +e_{1} P_{i} \Delta B_{i}(t) e_{2}^{T}+e_{2} \Delta B_{i}^{T}(t) P_{i} e_{1}^{T} .
\end{aligned}
$$

Considering (46) and combining the uncertainties condition (7) by Lemma 9 , we have

$$
\begin{gathered}
{\left[\begin{array}{cc}
\Pi_{i 1} & \Gamma^{T} M \\
M \Gamma & -M
\end{array}\right]+\left[\begin{array}{cc}
e_{1} P_{i} & 0 \\
M & 0
\end{array}\right] H_{i} F_{i}(t) \varepsilon^{T}} \\
+\varepsilon F_{i}^{T}(t) H_{i}^{T}\left[\begin{array}{cc}
P_{i} e_{1}^{T} & M \\
0 & 0
\end{array}\right]<0 .
\end{gathered}
$$

With (8), by Lemma 8 from (49), we obtain

$$
\begin{aligned}
& {\left[\begin{array}{cc}
\Pi_{i 1} & \Gamma^{T} M \\
M \Gamma & -M
\end{array}\right]+\frac{1}{\delta_{1}}\left[\begin{array}{cc}
e_{1} P_{i} & 0 \\
M & 0
\end{array}\right] H_{i} H_{i}^{T}\left[\begin{array}{cc}
P_{i} e_{1}^{T} & M \\
0 & 0
\end{array}\right]} \\
& +\delta_{1}\left[\begin{array}{rr}
\varepsilon \varepsilon^{T} & 0 \\
0 & 0
\end{array}\right]<0 .
\end{aligned}
$$


Obviously, (50) is equivalent to (44). Similarly, considering (46) and following the same procedure, we can get (45). Finally, following from the latter proof of Theorem 10, we know that the uncertain neutral systems with Markovian jump parameters and time-varying delay as described by (3) are stochastically stable. This completes the proof.

Remark 15. It should be noted that (44) and (45) can be viewed as linear matrix inequalities by introducing new variables. That is, define matrices

$$
\begin{aligned}
P_{i}^{(1)} & =\frac{1}{\delta_{1}} P_{i} H_{i} H_{i}^{T} P_{i}, & P_{i}^{(2)} & =\frac{1}{\delta_{2}} P_{i} H_{i} H_{i}^{T} P_{i}, \\
P_{i M}^{(1)} & =\frac{1}{\delta_{1}} P_{i} H_{i} H_{i}^{T} M, & P_{i M}^{(2)} & =\frac{1}{\delta_{2}} P_{i} H_{i} H_{i}^{T} M, \\
M^{(1)} & =\frac{1}{\delta_{1}} M H_{i} H_{i}^{T} M, & M^{(2)} & =\frac{1}{\delta_{2}} M H_{i} H_{i}^{T} M,
\end{aligned}
$$

where $H_{i}, i \in S$, are known constant matrices and have been defined in (7). Then (44) and (45) can be easily solved by the LMI Toolbox in MATLAB.

Remark 16. It should be mentioned that Theorem 14 is an extension of Theorem 10 to uncertain neutral Markovian jump systems with interval time-varying delays. It provides a stochastic delay-range-dependent stability criterion for (3) and it will be verified to be less conservative than some existing ones in Section 4.

Consistent with the nominal systems, in the uncertain case, we have the following result as a corollary if the information on the delay derivative $\mu$ may not be available. The corollary is also obtained by setting $R_{3}=0$ in Theorem 14 .

Corollary 17. For the given finite set $S$ of modes with transition rates matrix, scalars $d_{1}, d_{2}$, and $\tau$, the uncertain neutral systems with Markovian jump parameters and time-varying delays as described by (3) are stochastically stable if the operator D) is stable, and there exist scalars $\delta_{1}>0, \delta_{2}>0$, symmetric positive matrices $P_{i}>0(i \in S), Q_{j}>0(j=1,2,3,4,5)$, and $R_{k}>0(k=1,2,4,5, \ldots, 11)$ such that the following symmetric matrix inequalities hold:

$$
\begin{gathered}
{\left[\begin{array}{cc}
\widetilde{\Pi}_{i 1}+\frac{1}{\delta_{1}} e_{1} P_{i} H_{i} H_{i}^{T} P_{i} e_{1}^{T}+\delta_{1} \varepsilon \varepsilon^{T} & \left.\Gamma^{T}+\frac{1}{\delta_{1}} e_{1} P_{i} H_{i} H_{i}^{T}\right) M \\
* & \frac{1}{\delta_{1}} M H_{i} H_{i}^{T} M-M
\end{array}\right]<0,} \\
{\left[\begin{array}{cc}
\widetilde{\Pi}_{i 2}+\frac{1}{\delta_{2}} e_{1} P_{i} H_{i} H_{i}^{T} P_{i} e_{1}^{T}+\delta_{2} \varepsilon \varepsilon^{T} & \left(\Gamma^{T}+\frac{1}{\delta_{2}} e_{1} P_{i} H_{i} H_{i}^{T}\right) M \\
* & \frac{1}{\delta_{2}} M H_{i} H_{i}^{T} M-M
\end{array}\right]<0,}
\end{gathered}
$$

where $\widetilde{\Pi}_{i 1}$ and $\widetilde{\Pi}_{i 2}$ have been defined in Corollary 13 , the remaining notations are the same as Theorem 14.

\section{Numerical Examples}

In this section, numerical examples are given to show that the proposed theoretical results in this paper are effective and less conservative than some previous ones in the literature.

Example 1. Consider the nominal system in the form of (9) described as follows:

$$
\dot{x}(t)-C_{i} \dot{x}(t-0.1)=A_{i} x(t)+B_{i} x(t-d(t)),
$$

where $i \in S=\{1,2\}$ and the mode switching is governed by the rate matrix $\left[\pi_{i j}\right]_{2 \times 2}=\left[\begin{array}{cc}-5 & 5 \\ 4 & -4\end{array}\right]$, which is described by Figure 1

$$
\begin{aligned}
& A_{1}=\left[\begin{array}{cc}
2 & 5 \\
-2 & -3
\end{array}\right], \quad B_{1}=\left[\begin{array}{cc}
-0.3 & 0.5 \\
-0.2 & -0.3
\end{array}\right], \\
& C_{1}=\left[\begin{array}{cc}
-0.2 & 0 \\
0.1 & -0.2
\end{array}\right], \quad A_{2}=\left[\begin{array}{cc}
-5 & -1.6 \\
2 & -4
\end{array}\right] \text {, } \\
& B_{2}=\left[\begin{array}{cc}
-0.3 & 0.5 \\
-0.2 & -0.3
\end{array}\right], \quad C_{2}=\left[\begin{array}{cc}
-0.1 & 0.2 \\
0 & -0.2
\end{array}\right] \text {. }
\end{aligned}
$$

Given the time-varying delay $d(t)=0.5\left(2+\sin ^{3} t\right)$, from the graph of $d(t)$ with $t \in[0,2 \pi]$ in Figure 2 , we easily obtain $d_{1}=$ 0.5 and $d_{2}=1.5$. In addition, we have $\dot{d}(t)=1.5 \sin ^{2} t \cos t$ and its maximum $\mu=\sqrt{3} / 3$.

By Theorem 10, with the help of LMI toolbox in MAT$\mathrm{LAB}$, we solve (19) and get a group of matrices for the solution to guarantee the stochastic stability for the system (53) as follows: for simplicity, we only list the matrices for $P_{i}, i \in S=$ $\{1,2\}, Q_{j}, j=1,2, \ldots, 5$.

$$
\begin{aligned}
& P_{1}=\left[\begin{array}{cc}
1.4857 & -0.3614 \\
* & 0.6329
\end{array}\right], \quad P_{2}=\left[\begin{array}{cc}
2.0645 & -0.3761 \\
* & 0.5086
\end{array}\right], \\
& Q_{1}=\left[\begin{array}{cc}
0.7342 & -0.1546 \\
* & 0.2978
\end{array}\right], \quad Q_{2}=\left[\begin{array}{cc}
0.4083 & -0.1873 \\
* & 0.3652
\end{array}\right] \text {, } \\
& Q_{3}=\left[\begin{array}{cc}
0.4165 & -0.2137 \\
* & 0.3056
\end{array}\right], \quad Q_{4}=\left[\begin{array}{cc}
0.4576 & -0.2539 \\
* & 0.3684
\end{array}\right] \text {, } \\
& Q_{5}=\left[\begin{array}{cc}
0.2673 & -0.0845 \\
* & 0.1766
\end{array}\right] \text {. }
\end{aligned}
$$

Therefore, it can be seen that the system (9) is determined to be stochastically stable by Theorem 10 .

Example 2. As said in the literature [32], with the abrupt variation in its structures and parameters, we can present the partial element equivalent circuit (PEEC) model as a stochastic jump one. Then, a general form of PEEC model is given by (3), where we assume that the neutral delay of the PEEC model is constant. Consider the stochastic neutral partial element equivalent circuit (PEEC) model described by the following equation:

$$
\begin{aligned}
\dot{x}(t)-C_{i} \dot{x}(t-0.3)= & \left(A_{i}+\Delta A(t)\right) x(t) \\
& +\left(B_{i}+\Delta B(t)\right) x(t-d(t)),
\end{aligned}
$$




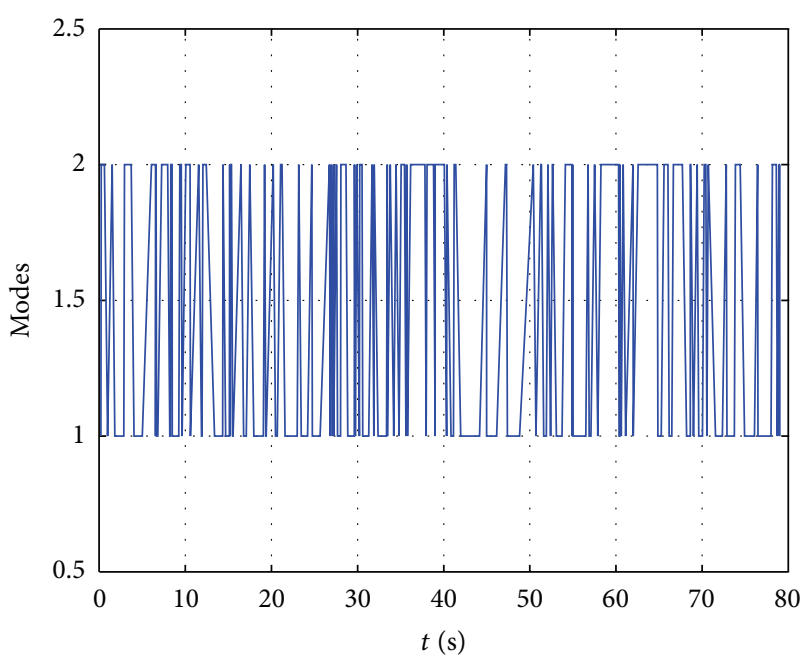

FIgURE 1: Operation modes of Example 1.

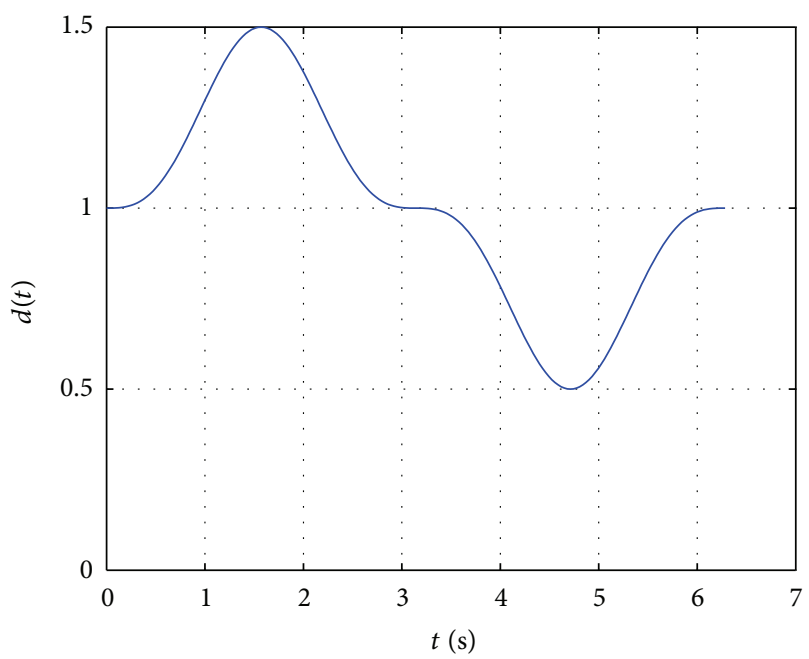

Figure 2: Interval time-varying delay $d(t)$ of Example 1.

where $i \in S=\{1,2\}$ and the mode switching is governed by the rate matrix $\left[\pi_{i j}\right]_{2 \times 2}=\left[\begin{array}{cc}-4 & 4 \\ 3 & -3\end{array}\right]$, which is described by Figure 3

$$
\begin{array}{cc}
A_{1}=\left[\begin{array}{cc}
-5 & 0 \\
0 & -6
\end{array}\right], & B_{1}=\left[\begin{array}{cc}
-1.6 & 0 \\
-1.8 & -1.5
\end{array}\right], \\
C_{1}=0.5 I, & H_{1}=\left[\begin{array}{l}
0.2 \\
0.2
\end{array}\right], \\
A_{2}=\left[\begin{array}{cc}
-4 & 0 \\
0 & -5
\end{array}\right], & B_{2}=\left[\begin{array}{cc}
-2 & 0 \\
-0.9 & -1.2
\end{array}\right], \\
C_{2}=0.3 I, & H_{2}=\left[\begin{array}{c}
0 \\
-0.3
\end{array}\right], \\
E_{A 1}=\left[\begin{array}{ll}
0.2 & 0
\end{array}\right], & E_{A 2}=\left[\begin{array}{ll}
0 & 0.2
\end{array}\right], \\
E_{B 1}=\left[\begin{array}{ll}
-0.3 & 0.3
\end{array}\right], & E_{B 2}=\left[\begin{array}{ll}
0.2 & 0.2
\end{array}\right] .
\end{array}
$$

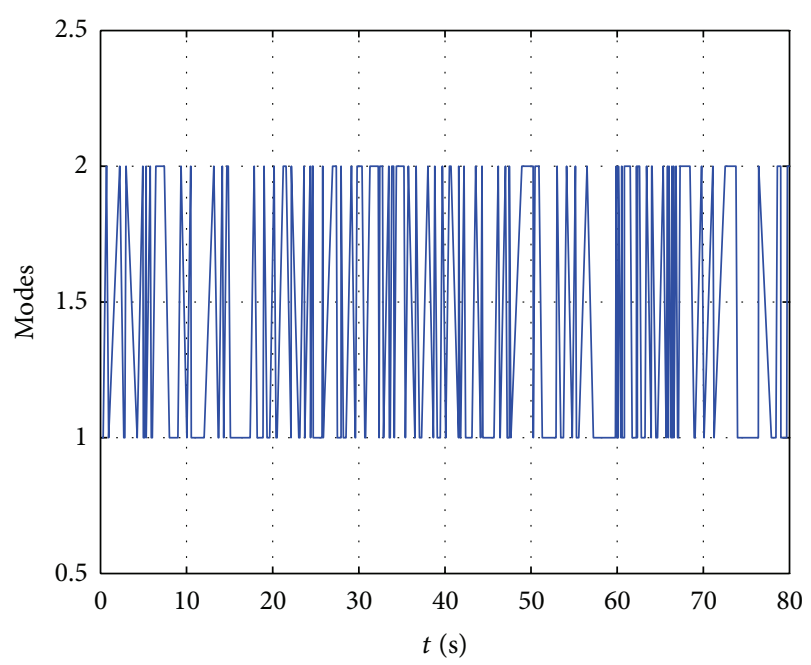

Figure 3: Operation modes of Example 2.

Given $\left\|F_{i}(t)\right\|<1$ and the time-varying delay $d(t)=0.5(2+$ $\cos ^{3} t$ ), from the graph of $d(t)$ with $t \in[0,2 \pi]$ in Figure 4, we easily obtain $d_{1}=0.5$ and $d_{2}=1.5$. In addition, we have $\dot{d}(t)=-1.5 \cos ^{2} t \sin t$ and its maximum $\mu=\sqrt{3} / 3$.

By Theorem 14, with the help of LMI toolbox in MAT$\mathrm{LAB}$, we solve (44) and (45) and get a group of matrices for the solution to guarantee the stochastic stability for the system (56) as follows: for simplicity, we only list the matrices for $P_{i}$, $i \in S=\{1,2\}, Q_{j}, j=1,2, \ldots, 5$.

$$
\begin{array}{cc}
P_{1}=\left[\begin{array}{cc}
2.4327 & -0.4713 \\
* & 0.7846
\end{array}\right], & P_{2}=\left[\begin{array}{cc}
2.7685 & -0.4617 \\
* & 0.7432
\end{array}\right], \\
Q_{1}=\left[\begin{array}{cc}
0.5122 & -0.1558 \\
* & 0.3976
\end{array}\right], & Q_{2}=\left[\begin{array}{cc}
0.6083 & -0.1898 \\
* & 0.3976
\end{array}\right], \\
Q_{3}=\left[\begin{array}{cc}
0.3164 & -0.2058 \\
* & 0.4751
\end{array}\right], & Q_{4}=\left[\begin{array}{cc}
0.2563 & -0.1584 \\
* & 0.3476
\end{array}\right], \\
Q_{5}=\left[\begin{array}{cc}
0.1574 & -0.0713 \\
* & 0.1798
\end{array}\right] .
\end{array}
$$

Therefore, according to Theorem 14, the uncertain neutral PEEC system presented by (3) is stochastically stable.

Example 3. In the study of practical electrical circuit systems, a small test circuit which consists of a partial element equivalent circuit (PEEC) was considered in [33], which can be described as the following form:

$$
\dot{x}(t)-C \dot{x}(t-\tau)=A x(t)+B x(t-d) .
$$

Compared with (9), (59) can be regarded as $i \in S=\{1\}$ and $d(t)=d$. So we have $d_{1}=d_{2}=d, \mu=0$ and utilize Theorem 10 to compute the maximum discrete delay for system stability. 


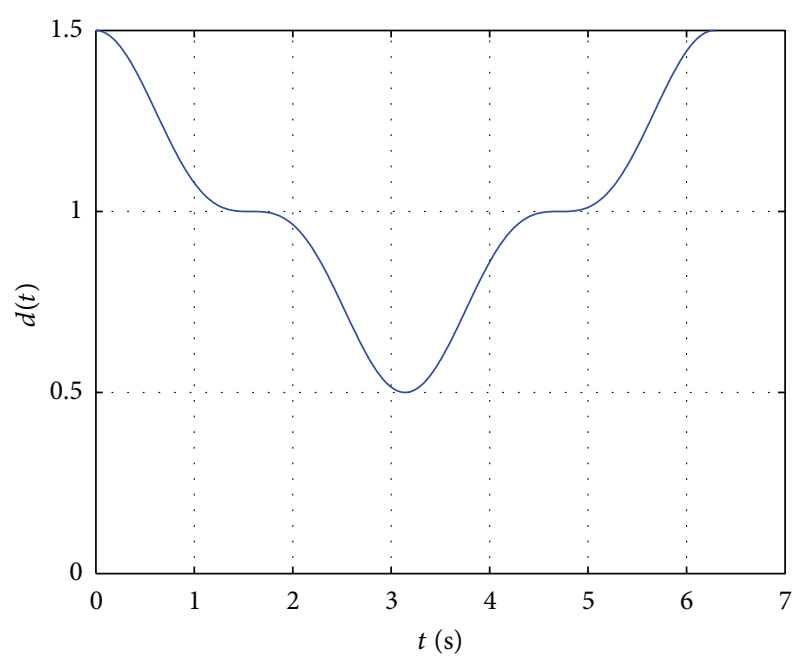

Figure 4: Interval time-varying delay $d(t)$ of Example 2.

Remark 18. It should be pointed out that we require $d_{1} \neq d_{2}$ and $d_{1} \neq 0$ in order to conveniently organize this paper. But from the results of the theorems and corollaries in this paper, we know that they are applicable to many special cases, such as $d(t) \equiv d, d_{1}=d_{2}$, or $d_{1}=0, \tau=0$. Actually, we just need to delete the corresponding integral terms in the Lyapunov functional and obtain homologous results.

Consider (59) with the following parameters:

$$
\begin{gathered}
A=\left[\begin{array}{cc}
-0.9 & 0.2 \\
0.1 & -0.9
\end{array}\right], \quad B=\left[\begin{array}{ll}
-1.1 & -0.2 \\
-0.1 & -1.1
\end{array}\right], \\
C=\left[\begin{array}{cc}
-0.2 & 0 \\
0.2 & -0.1
\end{array}\right] .
\end{gathered}
$$

For given $\tau$, by Theorem 10, the maximum $d$, which satisfies the LMIs in (19), can be calculated by solving a quasiconvex optimization problem. This neutral system was considered in references [34-36]. The results on the maximum upper bound of $d$ are compared in Table 1 .

From Table 1, we know that the maximum upper bound of delay $d=2.3026$ in this paper by setting $\tau=0.1$, while the maximum upper bound of delay $d=1.7100$ for [36], $d=$ 2.1229 for [34], and $d=2.2951$ for [35]. The results are also given by setting $\tau=0.5$ and $\tau=1$, and it is found that the maximum upper bound in this paper is larger than those in [34-36]. So it can be demonstrated that the stability condition Theorem 10 in this paper yields less conservative results than the previous ones.

Example 4. Further consideration on Example 3, if we take the parameter uncertainties commonly existing in the modeling of a real system into account, a general form of PEEC model is given by

$$
\begin{aligned}
\dot{x}(t)-C \dot{x}(t-\tau)= & (A+\Delta A(t)) x(t) \\
& +(B+\Delta B(t)) x(t-d),
\end{aligned}
$$

TABLE 1: Maximum upper bound of $d$ with different neutral delay $\tau$.

\begin{tabular}{lccc}
\hline Methods & $\tau=0.1$ & $\tau=0.5$ & $\tau=1$ \\
\hline He et al. [36] & 1.7100 & 1.6718 & 1.6543 \\
Han [34] & 2.1229 & 2.1229 & 2.1229 \\
Li et al. [35] & 2.2951 & 2.3471 & 2.3752 \\
Theorem 10 & 2.3026 & 2.3547 & 2.3835 \\
\hline
\end{tabular}

where $A, B$, and $C$ are given in Example 3 and the uncertain matrices $\Delta A(t)$ and $\Delta B(t)$ satisfy

$$
\|\Delta A(t)\| \leq \kappa, \quad\|\Delta B(t)\| \leq \kappa, \quad \kappa \geq 0 .
$$

Moreover, in the form of (7) and (8), we assume that

$$
H=\kappa I, \quad E_{A}=E_{B}=\mathrm{I}, \quad 0 \leq \kappa \leq 1 .
$$

Consider (61), for given $\tau$ and $\kappa$, by Theorem 14, the maximum upper bound of $d$, which satisfies the LMIs in (44) and (45), can be calculated by solving a quasiconvex optimization problem. When $\tau=1.0$, Table 2 gives the comparisons of the maximum allowed delay of $d$ for various parameters $\kappa$ in different methods.

From Table 2, provided that $\tau=1.0$, we know that the maximum upper bound of delay $d=1.5316$ in this paper by setting $\kappa=0.10$, while the maximum upper bound of delay $d=1.3864$ for [37], $d=1.4385$ for [36], and $d=1.5047$ for [38]. The results are also given by setting $\kappa=0.15, \kappa=0.20$, and $\kappa=0.25$, and it is found that the maximum upper bound in this paper is larger than those in [36-38]. So it can be seen that the delay-range-dependent stability condition Theorem 14 in this paper is less conservative than some earlier reported ones in the literature.

Example 5. In this example, to compare the stochastic stability result in Theorem 10 with those in $[23,39,40]$, we consider the nominal system (9) with $C_{r_{t}}=0$ and $d_{1}=0$. In fact, we consider here that there are no longer neutral delay systems, for given (9) with the following parameters:

$$
\begin{gathered}
A_{1}=\left[\begin{array}{cc}
-3.49 & 0.81 \\
-0.65 & -3.27
\end{array}\right], \quad A_{2}=\left[\begin{array}{cc}
-2.49 & 0.29 \\
1.34 & -0.02
\end{array}\right], \\
B_{1}=\left[\begin{array}{cc}
-0.86 & -1.29 \\
-0.68 & -2.07
\end{array}\right], \quad B_{2}=\left[\begin{array}{cc}
-2.83 & 0.50 \\
-0.84 & -1.01
\end{array}\right], \\
C_{1}=C_{2}=0, \\
P_{i j}=\left[\pi_{i j}\right]_{2 \times 2}, \quad i, j \in S=\{1,2\} .
\end{gathered}
$$

As described previously, for given $\pi_{22}=-0.8$, different values of $\pi_{11}$ and different values of $\mu$, by Theorem 10 and the maximum $d_{2}$, which satisfies the LMIs in (19), can be calculated by solving a quasiconvex optimization problem. Tables 3 and 4 give the contrastive results.

From Table 3, provided that $\mu=0$, we know that the maximum upper bound of delay $d_{2}=0.6853$ in this paper by setting $\pi_{11}=-0.10$, while the maximum upper bound of delay $d_{2}=0.5012$ for [40], $d_{2}=0.5012$ for [39], and 
TABLE 2: Maximum upper bound of $d$ with $\tau=1.0$ and different parameter $\kappa$.

\begin{tabular}{lcccc}
\hline Methods & $\kappa=0.10$ & $\kappa=0.15$ & $\kappa=0.20$ & $\kappa=0.25$ \\
\hline Han [37] & 1.3864 & 1.2705 & 1.1607 & 1.0456 \\
He et al. [36] & 1.4385 & 1.3309 & 1.2396 & 1.1547 \\
Xu et al. [38] & 1.5047 & 1.4052 & 1.2998 & 1.2136 \\
Theorem 14 & 1.5316 & 1.4089 & 1.3028 & 1.2217 \\
\hline
\end{tabular}

TABLE 3: Maximum upper bound of $d_{2}$ with $\mu=0$ and different parameter $\pi_{11}$.

\begin{tabular}{lcccc}
\hline Methods & $\pi_{11}=-0.10$ & $\pi_{11}=-0.50$ & $\pi_{11}=-0.80$ & $\pi_{11}=-1.00$ \\
\hline Cao et al. [40] & 0.5012 & 0.4941 & 0.4915 & 0.4903 \\
Chen et al. [39] & 0.5012 & 0.4941 & 0.4915 & 0.4903 \\
Xu et al. [23] & 0.6797 & 0.5794 & 0.5562 & 0.5465 \\
Theorem 10 & 0.6853 & 0.5874 & 0.5625 & 0.5574 \\
\hline
\end{tabular}

TABLE 4: Maximum upper bound of $d_{2}$ with $\mu=1.5$ and different parameter $\pi_{11}$.

\begin{tabular}{lcccc}
\hline Methods & $\pi_{11}=-0.10$ & $\pi_{11}=-0.50$ & $\pi_{11}=-0.80$ & $\pi_{11}=-1.00$ \\
\hline Cao et al. [40] & - & - & - & - \\
Chen et al. [39] & - & - & - & - \\
Xu et al. [23] & 0.3860 & 0.3656 & 0.3487 & 0.3378 \\
Theorem 10 & 0.3953 & 0.3746 & 0.3502 & 0.3449 \\
\hline
\end{tabular}

$d_{2}=0.6797$ for [23]. The results are also given by setting $\pi_{11}=-0.50, \pi_{11}=-0.80$, and $\pi_{11}=-1.00$, and it is found that the maximum upper bound of delay $d_{2}$ in this paper is larger than those in $[23,39,40]$. So it also can be shown that the stochastic stability result in Theorem 10 is less conservative than those results in $[23,39,40]$.

From Table 4, provided that $\mu=1.5$, we know that the maximum upper bound of delay $d_{2}=0.3953$ in this paper by setting $\pi_{11}=-0.10$, while the methods in $[39,40]$ cannot be applicable to the case $\mu \geq 1$, and the maximum upper bound of delay $d_{2}=0.3860$ for [23]. So it can be shown that Theorem 10 in this paper is less conservative and can be applied to the time-varying delay without the requirement on $\mu<1$.

\section{Conclusions}

In this paper, some new delay-range-dependent conditions have been provided to guarantee the stochastic stability of the neutral systems with Markovian jumping parameters and interval time-varying delays. A novel augmented LyapunovKrasovskii functional which contains some triple-integral terms is constructed. By some integral inequalities and the nature of convex combination, some less conservative delayrange-dependent stochastic stability criteria are obtained. Numerical examples are given to demonstrate the effectiveness and less conservativeness of our result.

\section{Acknowledgments}

This work was supported in part by the National Key Scientific Research Project (61233003), the National Natural Science Foundation of China (60935001, 61174061, 61074033, and 60934006), and the Fundamental Research Funds for the Central Universities.

\section{References}

[1] Y. Kuang, Delay Differential Equations with Applications in Population Dynamics, vol. 191 of Mathematics in Science and Engineering, Academic Press, Boston, Mass, USA, 1993.

[2] Q. Zhang, L. Yang, and J. Liu, "Dynamics of fuzzy BAM neural networks with distributed delays and diffusion," Journal of Applied Mathematics, vol. 2012, Article ID 136048, 19 pages, 2012.

[3] S.-I. Niculescu, Delay Effects on Stability: A Robust Control Approach, vol. 269 of Lecture Notes in Control and Information Sciences, Springer, London, UK, 2001.

[4] J. K. Hale and S. M. Verduyn Lunel, Introduction to FunctionalDifferential Equations, vol. 99 of Applied Mathematical Sciences, Springer, New York, NY, USA, 1993.

[5] V. B. Kolmanovskii and A. D. Myshkis, Theory of Functional Differential Equations, Kluwer Academic, Dordrecht, The Netherlands, 1992.

[6] A.-G. Wu and G.-R. Duan, "On delay-independent stability criteria for linear time-delay systems," International Journal of Automation and Computing, vol. 4, no. 1, pp. 95-100, 2007.

[7] E. K. Boukas and N. F. Al-Muthairi, "Delay-dependent stabilization of singular linear systems with delays," International Journal of Innovative Computing, Information and Control, vol. 2, no. 2, pp. 283-291, 2006.

[8] P. L. Liu, "Further results on the exponential stability criteria for time delay singular systems with delay-dependence," International Journal of Innovative Computing, Information and Control, vol. 8, no. 6, pp. 4015-4024, 2012.

[9] U. Başer and U. Şahin, "Improved delay-dependent robust stabilization of singular systems," International Journal of Innovative Computing, Information and Control, vol. 7, no. 1, pp. 177-187, 2011.

[10] C.-C. Shen and S.-M. Zhong, "New delay-dependent robust stability criterion for uncertain neutral systems with timevarying delay and nonlinear uncertainties," Chaos, Solitons and Fractals, vol. 40, no. 5, pp. 2277-2285, 2009.

[11] C.-H. Lien and J.-D. Chen, "Discrete-delay-independent and discrete-delay-dependent criteria for a class of neutral systems," Journal of Dynamic Systems, Measurement and Control, Transactions of the ASME, vol. 125, no. 1, pp. 33-41, 2003.

[12] H. R. Karimi, M. Zapateiro, and N. Luo, "Stability analysis and control synthesis of neutral systems with time-varying delays and nonlinear uncertainties," Chaos, Solitons and Fractals, vol. 42, no. 1, pp. 595-603, 2009.

[13] J. H. Park, "Novel robust stability criterion for a class of neutral systems with mixed delays and nonlinear perturbations," Applied Mathematics and Computation, vol. 161, no. 2, pp. 413421, 2005.

[14] M. N. A. Parlakçı, "Delay-dependent robust stability criteria for uncertain neutral systems with mixed time-varying discrete and neutral delays," Asian Journal of Control, vol. 9, no. 4, pp. 411421, 2007. 
[15] O. M. Kwon and J. H. Park, "Delay-range-dependent stabilization of uncertain dynamic systems with interval time-varying delays," Applied Mathematics and Computation, vol. 208, no. 1, pp. 58-68, 2009.

[16] S. Xu and J. Lam, "A survey of linear matrix inequality techniques in stability analysis of delay systems," International Journal of Systems Science, vol. 39, no. 12, pp. 1095-1113, 2008.

[17] B. Chen, H. Li, P. Shi, C. Lin, and Q. Zhou, "Delay-dependent stability analysis and controller synthesis for Markovian jump systems with state and input delays," Information Sciences, vol. 179, no. 16, pp. 2851-2860, 2009.

[18] E. K. Boukas, Z. K. Liu, and P. Shi, “Delay-dependent stability and output feedback stabilisation of Markov jump system with time-delay," IEE Proceedings, vol. 149, no. 5, pp. 379-386, 2002.

[19] J. Liu, Z. Gu, and S. Hu, " $H_{\infty}$ filtering for Markovian jump systems with time-varying delays," International Journal of Innovative Computing, Information and Control, vol. 7, no. 3, pp. 1299-1310, 2011.

[20] J. Wu, T. Chen, and L. Wang, "Delay-dependent robust stability and $H_{\infty}$ control for jump linear systems with delays," Systems \& Control Letters, vol. 55, no. 11, pp. 939-948, 2006.

[21] P. Balasubramaniam, A. Manivannan, and R. Rakkiyappan, "Exponential stability results for uncertain neutral systems with interval time-varying delays and Markovian jumping parameters," Applied Mathematics and Computation, vol. 216, no. 11, pp. 3396-3407, 2010.

[22] P. Shi, Y. Xia, G. P. Liu, and D. Rees, "On designing of slidingmode control for stochastic jump systems," Institute of Electrical and Electronics Engineers, vol. 51, no. 1, pp. 97-103, 2006.

[23] S. Xu, J. Lam, and X. Mao, "Delay-dependent $H_{\infty}$ control and filtering for uncertain Markovian jump systems with timevarying delays," IEEE Transactions on Circuits and Systems, vol. 54, no. 9, pp. 2070-2077, 2007.

[24] Q.-L. Han, "Robust stability of uncertain delay-differential systems of neutral type," Automatica, vol. 38, no. 4, pp. 719-723, 2002.

[25] Y. Zhang, Y. He, M. Wu, and J. Zhang, "Stabilization for Markovian jump systems with partial information on transition probability based on free-connection weighting matrices," Automatica, vol. 47, no. 1, pp. 79-84, 2011.

[26] X. Mao, "Stability of stochastic differential equations with Markovian switching," Stochastic Processes and their Applications, vol. 79, no. 1, pp. 45-67, 1999.

[27] K. Gu, "An improved stability criterion for systems with distributed delays," International Journal of Robust and Nonlinear Control, vol. 13, no. 9, pp. 819-831, 2003.

[28] J. Sun, G. P. Liu, and J. Chen, "Delay-dependent stability and stabilization of neutral time-delay systems," International Journal of Robust and Nonlinear Control, vol. 19, no. 12, pp. 13641375, 2009.

[29] L. Xie, "Output feedback $H_{\infty}$ control of systems with parameter uncertainty," International Journal of Control, vol. 63, no. 4, pp. 741-750, 1996.

[30] H. B. Ji, Algebra Foundation of Control Theory, University of Science and Technology of China Press, Hefei, China, 2008.

[31] F. C. Klebaner, Introduction to Stochastic Calculus with Applications, Imperial College Press, London, UK, 2nd edition, 2005.

[32] S. He and F. Liu, "Exponential stability for uncertain neutral systems with Markov jumps," Journal of Control Theory and Applications, vol. 7, no. 1, pp. 35-40, 2009.
[33] A. Bellen, N. Guglielmi, and A. E. Ruehli, "Methods for linear systems of circuit delay differential equations of neutral type," IEEE Transactions on Circuits and Systems, vol. 46, no. 1, pp. 212215, 1999.

[34] Q.-L. Han, "On stability of linear neutral systems with mixed time delays: a discretized Lyapunov functional approach," Automatica, vol. 41, no. 7, pp. 1209-1218, 2005.

[35] X.-G. Li, X.-J. Zhu, A. Cela, and A. Reama, "Stability analysis of neutral systems with mixed delays," Automatica, vol. 44, no. 11, pp. 2968-2972, 2008.

[36] Y. He, M. Wu, J.-H. She, and G.-P. Liu, "Delay-dependent robust stability criteria for uncertain neutral systems with mixed delays," Systems \& Control Letters, vol. 51, no. 1, pp. 57-65, 2004.

[37] Q. L. Han, "A descriptor system approach to robust stability of uncertain neutral systems with discrete and distributed delays," in Proceedings of the American Control Conference, pp. 50985103, Denver, June 2003.

[38] S. Xu, J. Lam, and Y. Zou, "Further results on delay-dependent robust stability conditions of uncertain neutral systems," International Journal of Robust and Nonlinear Control, vol. 15, no. 5, pp. 233-246, 2005.

[39] W. H. Chen, Z. H. Guan, and X. Lu, "Delay-dependent output feedback stabilisation of Markovian jump system with timedelay," IEE Proceedings-Control Theory and Applications, vol. 151, pp. 561-566, 2004.

[40] Y.-Y. Cao, W. Yan, and A. Xue, "Improved delay-dependent stability conditions and Ho control for jump time-delay systems," in Proceedings of the 43rd IEEE Conference on Decision and Control (CDC '04), pp. 4527-4532, December 2004. 


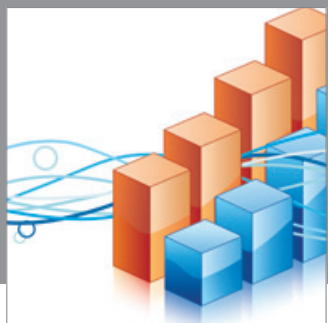

Advances in

Operations Research

mansans

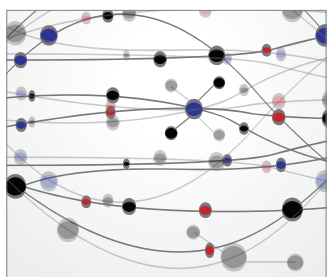

The Scientific World Journal
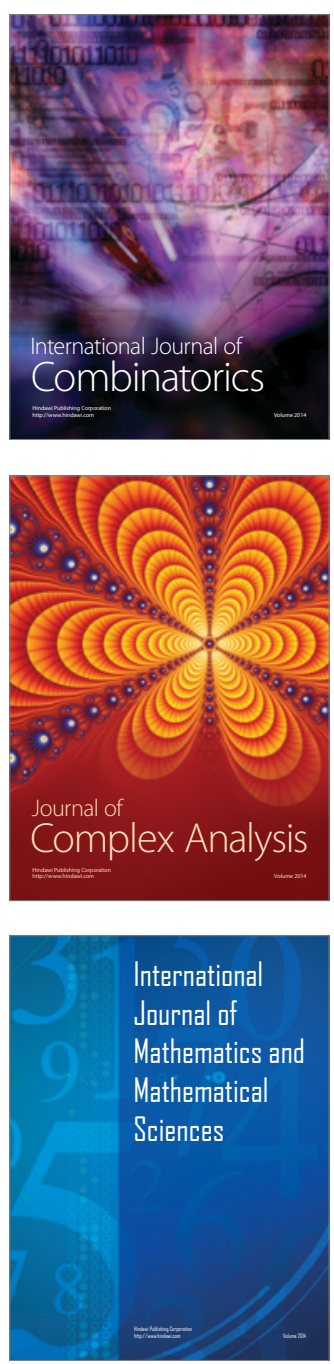
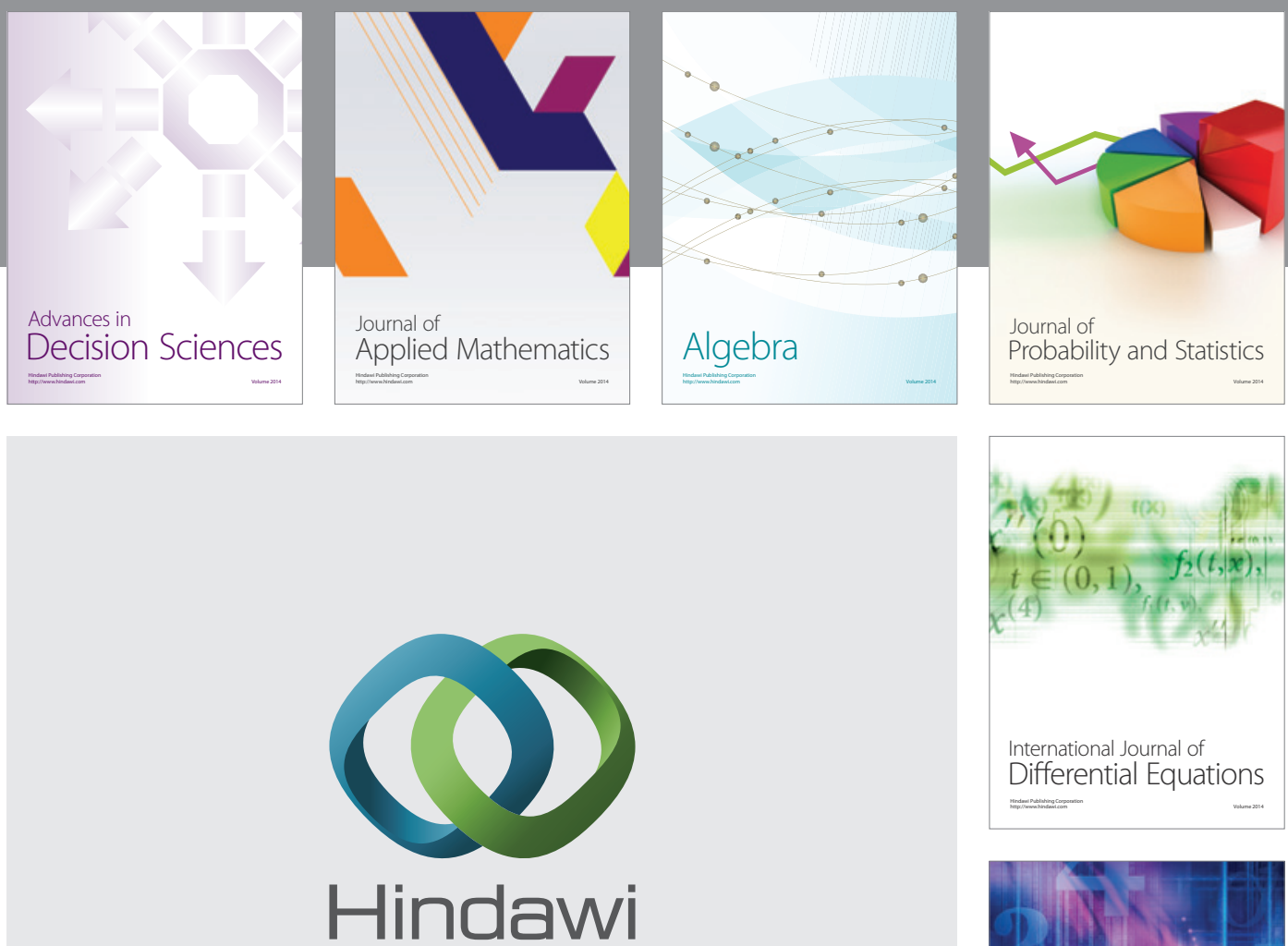

Submit your manuscripts at http://www.hindawi.com
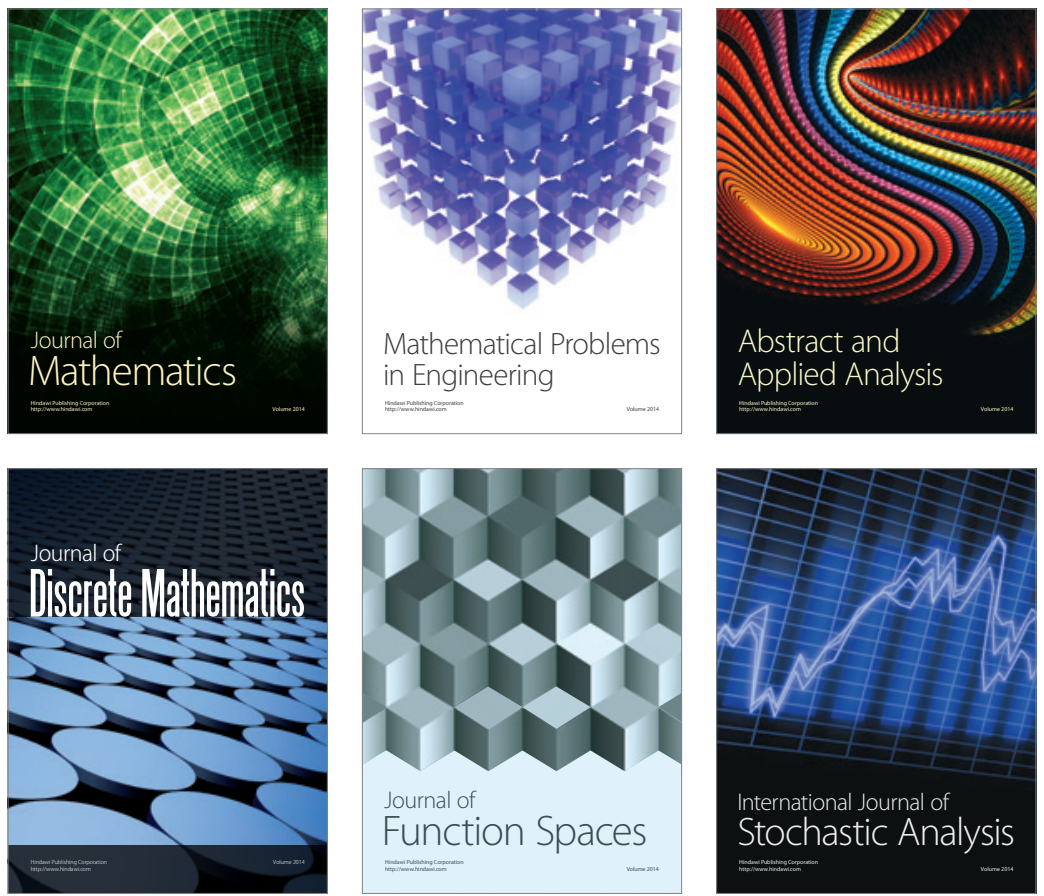

Journal of

Function Spaces

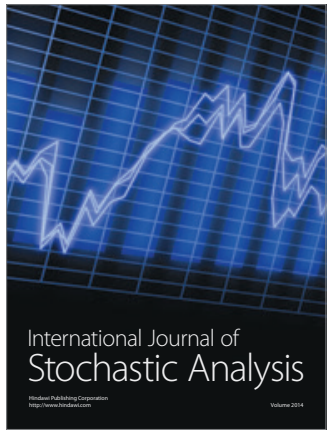

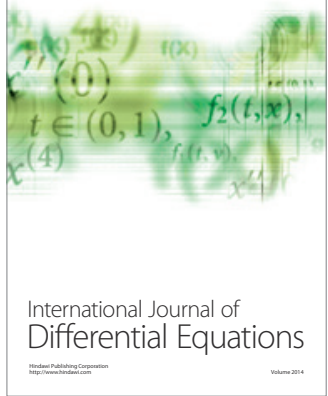
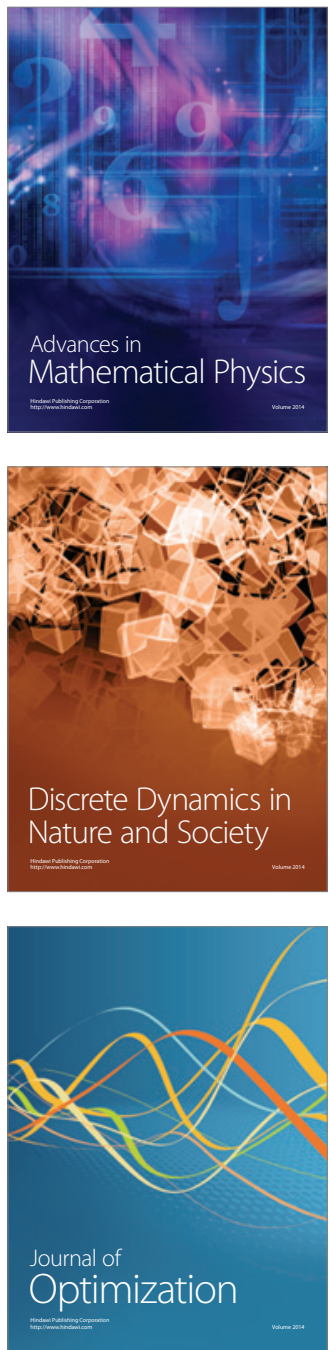